\title{
THE $K$-THEORY OF TRIANGULAR MATRIX RINGS. II
}

\author{
M. E. KEATING
}

\begin{abstract}
Let $T$ be the upper triangular matrix ring defined by a pair of rings $R$ and $S$ and an $R$ - $S$-bimodule $M$. We use the "QP" definition of algebraic $K$-theory to give a quick proof that the homomorphism
\end{abstract}

$$
\pi_{m}: K_{m}(T) \rightarrow K_{m}(R) \oplus K_{m}(S), \quad m \geqslant 0,
$$

induced by the obvious ring epimorphism is an isomorphism.

Given rings $R$ and $S$ and an $R$-S-bimodule $M$, one can construct an upper triangular matrix ring

$$
T=\left[\begin{array}{cc}
R & M \\
0 & S
\end{array}\right],
$$

with the evident addition and multiplication.

Let $\pi: T \rightarrow R \oplus S$ be the natural ring epimorphism and let

$$
\pi_{m}: K_{m}(T) \rightarrow K_{m}(R) \oplus K_{m}(S), \quad m \in \mathbf{Z},
$$

be the induced homomorphism in $K$-theory.

It is well known that $\pi_{0}$ and $\pi_{1}$ are isomorphisms and Dennis and Geller [2] showed that $\pi_{2}$ is also an isomorphism. In a recent paper [1], Jon Berrick and myself confirmed the conjecture that all the $\pi_{m}$ are in fact isomorphisms. The arguments in both papers were based on the " $\mathrm{BGL}^{+}$" definition of $K$-theory, requiring calculations in the homology of the general linear group.

The purpose of this note is to present an alternative proof of the conjecture, using the "QP" definition of $K$-theory and the calculus of functors on the category $\mathbf{P}(T)$ of finitely generated projective left $T$-modules. A similar argument was used in [3], but there I relied on homological conditions on the bimodule $M$ which were natural for the rings under investigation.

We consider only the case $m \geqslant 0$.

Let $\rho$ be $\pi$ followed by projection onto the first factor of $R \oplus S$ and write

$$
A=\left[\begin{array}{cc}
0 & M \\
0 & S
\end{array}\right]
$$

so that there is an exact sequence of rings and ideals

$$
0 \rightarrow A \rightarrow T \stackrel{\rho}{\rightarrow} R \rightarrow 0 \text {. }
$$

Received by the editors November 4, 1985.

1980 Mathematics Subject Classification (1985 Revision). Primary 18F25, 16A42.

Key words and phrases. Algebraic $K$-theory, triangular matrix ring. 
Regard this sequence first as a sequence of left $T$-modules. The $T$-module structure induced on $R$ via $\rho$ is the same as that arising through the column $\left[\begin{array}{l}R \\ 0\end{array}\right]$; thus the sequence is split and both $R$ and $A$ are projective left $T$-modules. We can therefore define endofunctors $a$ and $b$ of $\mathbf{P}(T)$ by $a=A \otimes_{T}-$ and $b=R \otimes_{T}-$.

The following equivalences are easily verified:

$$
a^{2} \simeq a, \quad b^{2} \simeq b, \quad a b \simeq 0 \simeq b a .
$$

The functor $a \oplus b$ is not the identity functor on $\mathbf{P}(T)$, since it has the wrong effect on homomophisms, but if we think of the exact sequence above as a sequence of right modules, we see that there is an exact sequence

$$
0 \rightarrow a \rightarrow \mathrm{Id} \rightarrow b \rightarrow 0
$$

of exact functors on $\mathbf{P}(T)$.

Write $\alpha_{m}$ and $\beta_{m}$ for the endomorphisms of $K_{m}(T)$ induced by the functors $a$ and $b$ respectively. A fundamental result of Quillen [4, §3, Corollary 3] shows that

$$
1=\alpha_{m}+\beta_{m}
$$

on $K_{m}(T)$. Thus $\alpha_{m}$ and $\beta_{m}$ are mutually orthogonal idempotent endomorphisms of $K_{m}(T)$.

To see that the corresponding direct decomposition of $K_{m}(T)$ gives the promised isomorphism, we introduce the functors

and

$$
\begin{aligned}
& \left.r=R \otimes_{T}-: \mathbf{P}(T) \rightarrow \mathbf{P}(R) \quad \text { (induced by } \rho\right), \\
& w=\text { incl: } \mathbf{P}(R) \rightarrow \mathbf{P}(T), \\
& s=S \otimes_{T}-: \mathbf{P}(T) \rightarrow \mathbf{P}(S) \quad \text { (induced by projection to } S \text { ), } \\
& x=A \otimes_{S}-: \mathbf{P}(S) \rightarrow \mathbf{P}(T) .
\end{aligned}
$$

There are natural equivalences

$$
w r \simeq b, \quad r w \simeq \operatorname{Id}_{\mathbf{P}(R)}, \quad x s \simeq a \quad \text { and } \quad s x \simeq \operatorname{Id}_{\mathbf{P}(S)},
$$

corresponding to which there are identities on $K$-theory:

$$
\omega_{m} \rho_{m}=\beta_{m}, \quad \rho_{m} \omega_{m}=1 \text { on } K_{m}(R)
$$

and

$$
\xi_{m} \sigma_{m}=\alpha_{m}, \quad \sigma_{m} \xi_{m}=1 \text { on } K_{m}(S) .
$$

Since $\rho_{m} \oplus \sigma_{m}=\pi_{m}$, the result is proved.

\section{REFERENCES}

1. A. J. Berrick and M. E. Keating, The K-theory of triangular matrix rings, Applications of Algebraic $K$-theory to Algebraic Geometry and Number Theory, Contemp. Math., vol. 55, part I, Amer. Math. Soc., Providence, R. I., 1986, pp. 69-74.

2. R. K. Dennis and S. C. Geller, $K_{i}$ of upper triangular matrix rings, Proc. Amer. Math. Soc. 56 (1976), 73-78.

3. M. E. Keating, The $K$-theory of triangular matrix rings and orders, Algebraic $K$-theory, Number Theory, Geometry and Analysis, Bielefeld 1982, Lecture Notes in Math., vol. 1046, Springer, Berlin, and New York, 1984, pp. 178-192.

4. D. Quillen, Higher algebraic K-theory I, Algebraic $K$-theory 1, Batelle Institute Conference 1972, Lecture Notes in Math., vol. 341, Springer, Berlin, and New York, 1973, pp. 85-147.

Department of Mathematics, Imperial College, London SW7 2BZ, England 